\title{
Relaciones entre el sueño y la adicción
}

\section{Relationships between sleep and addiction}

\author{
| Francesca Cañellas*; Luis de Lecea*
}

\author{
* Servicio de Psiquiatría. Hospital Universitario Son Espases. Palma de \\ Mallorca. España. \\ ** Professor Dept of Psychiatry and Behavioral Sciences Stanford University \\ School of Medicine, California USA
}

Enviar correspondencia a:

Francesca Cañellas

Servei de Psiquiatria. Hospital Universitari Son Espases

CNalldemosa $n^{\circ}$ 79. 07010 Palma de Mallorca. España

francesca.canellas@ssib.es - Tel: +34871206673

\section{Resumen}

La interacción entre los trastornos del sueño y el abuso de sustancias es ya conocida, pero seguramente más compleja de lo que se pensaba. Existe tanto una relación positiva entre tener un trastorno por uso de substancias y sufrir un trastorno de sueño, como viceversa. Los efectos sobre el sueño dependen de la substancia utilizada, pero se ha demostrado que tanto durante su uso como en período de abstinencia los consumidores tienen diferentes problemas de sueño y fundamentalmente un sueño más fragmentado. Sabemos que hay que tener en cuenta los problemas de sueño para evitar recaídas en la adicción.

Investigaciones recientes indican que el sistema hipocretinérgico definido por el neuropéptido hipocretina/orexina (Hcrt/ox), localizado en el hipotálamo lateral e implicado entre otros en la regulación del ciclo sueñovigilia, jugaria un papel importante en las conductas adictivas. Diferentes estudios han demostrado interacciones entre el sistema hipocretinérgico, los circuitos de respuesta aguda al estrés y los sistemas de recompensa. También sabemos que la activación optogenética selectiva del sistema hipocretinérgico incrementa la probabilidad de la transición del sueño a la vigilia, y también es suficiente para iniciar un comportamiento compulsivo de recaída adictiva. La activación del sistema hipocretinérgico podria explicar la hipervigilia asociada al estrés y a la adicción. El mayor conocimiento de esta interacción permitiría entender mejor los mecanismos de la adicción y encontrar nuevas estrategias para el tratamiento de las adicciones.

Palabras clave: Sueño, adicción, estrés, hipervigilia, hipocretina/orexina (Hcrt/ox)

\section{Abstract}

While it is well known that there is an interaction between sleep disorders and substance abuse, it is certainly more complex than was previously thought. There is a positive relationship both between having a substance use disorder and suffering from a sleep disorder, and vice versa. The effects on sleep depend on the substance used, but it has been shown that both during use and in withdrawal periods consumers have various sleep problems, and basically more fragmented sleep. We know that sleep problems must be taken into account to prevent addiction relapses.

Recent research shows that the hypocretinergic system defined by neuropeptide hypocretin / orexin (Hcrt / ox), located in the lateral hypothalamus and involved in, among other things, the regulation of the sleep-wake cycle, may play an important role in addictive behaviors. Different studies have demonstrated interactions between the hypocretinergic system, acute response to stress circuits and reward systems. We also know that selective optogenetic activation of the hypocretinergic system increases the probability of transition from sleep to wakefulness, and is sufficient for initiating an addictive compulsive behavior relapse. Hypocretinergic system activation could explain the hyperarousal associated with stress and addiction. Improved knowledge of this interaction would help us to understand better the mechanisms of addiction and find new strategies for the treatment of addictions.

Key words: sleep, addiction, stress, hyperarousal, hypocretinergic system 
a interacción entre los trastornos del sueño y el abuso de sustancias es compleja. Hasta hace poco conocíamos una relación bidireccional positiva entre el riesgo de tener un trastorno por uso de substancias y sufrir un trastorno de sueño: las personas con abuso o dependencia de sustancias tienen mayor prevalencia de alteraciones del sueño y las que presentan alteraciones del sueño tienen mayor riesgo de presentar problemas relacionados con el uso de sustancias que la población general (Cañellas et al., 1998; Ford y Kamerow, 1989).

Investigaciones recientes apuntan que la interacción entre adicciones y niveles de vigilancia es más compleja. El ciclo vigilia sueño esta regulado por un conjunto de redes neuronales que controlan diferentes aspectos de la transición entre los diferentes estados de vigilancia. En concreto, el sistema definido por el neuropéptido hipocretina/orexina (Hcrt/ox), localizado en el hipotálamo lateral (de Lecea et al., 1998; Sakurai et al., 1998), juega un papel crucial en la estabilidad del ciclo, ya que su deficiencia da lugar a la narcolepsia con cataplejía (Hungs y Mignot 2001). El sistema Hcrt está implicado en el control de numerosas variables, como el estado metabólico, ritmo circadiano, duración previa del sueño, niveles de estrés, etc. y transmite esta información de forma integrada a otros circuitos esenciales para el mantenimiento de la vigilia (Sutcliffe y de Lecea, 2002).

La implicación tan significativa del sistema hipocretinérgico en la vigilia llevó a la hipótesis de un posible papel de este circuito en la hipervigilia asociada al estrés y la adicción, como describiremos más adelante.

\section{Acción de las principales drogas sobre el sueño}

En el estudio ECA (Ford y Kamerow, 1989) se describió que los pacientes que referían preocupaciones sobre el sueño tenían un riesgo relativo 2.32 veces mayor de desarrollar problemas relacionados con el alcohol, detectándose especialmente en los que presentaban historia de ansiedad y disforia. Aunque también se ha descrito esta relación con otras sustancias, la mayoría de los estudios han sido realizados en alcohólicos (Irwin et al. 2006). El insomnio, en la adolescencia, está relacionado con el desarrollo posterior de trastornos mentales, asociándose al uso de alcohol, cannabis, otras drogas, depresión y a ideas e intentos de suicidio. Además, el insomnio es más frecuente en los adolescentes, que fuman, beben y duermen menos tiempo durante el fin de semana (Roane et al., 2008).

Aunque las acciones sobre el sueño dependen de la droga consumida, y el objetivo de esta editorial no es repasar detalladamente cuales son específicamente estas alteraciones, nos detendremos a señalar algunas que son características y frecuentes, debido a su importancia en el manejo de los pacientes con trastorno del uso de substancias.

La mayoría de estudios se han realizado con el alcohol, que es el ansiolítico e hipnótico más ampliamente utilizado en el mundo occidental desde hace milenios. En consumo agudo esta sustancia ejerce un efecto inductor del sueño, pero en consumo continuado deteriora su calidad, problema que se agrava todavía más durante el periodo de abstinencia. A dosis bajas, como podría ser el consumo social reconocido como "normal" en los países latinos, con unos niveles plasmáticos de alcohol bajos, no tiene claros efectos sobre la arquitectura del sueño. A dosis más altas produce un efecto similar al de un hipnótico de acción corta: disminuye la latencia de sueño, así como los despertares, la fase de sueño 1 y la densidad de movimientos oculares rápidos (REM) en la primera mitad de la noche; mientras que en la segunda mitad se aprecian fenómenos de rebote con aumento de la fase de sueño 1, aumento de despertares y un incremento de sueño REM. En pacientes alcohólicos crónicos, se ha encontrado una disminución del sueño lento profundo, del sueño REM y un sueño más fragmentado. Durante la fase de abstinencia estas alteraciones se agravan. Los sujetos duermen menos, el sueño está más fragmentado y tienen una disminución del sueño lento, mientras que la duración del REM y la densidad de los movimientos oculares rápidos están incrementadas (Brower et al., 2011). De hecho, uno de los factores de recaída en el consumo de alcohol, tras un periodo de abstinencia puede ser la persistencia de alteraciones del sueño. Por ello se recomienda dar inductores del sueño durante la fase de abstinencia aguda y, si el insomnio persiste, utilizar antidepresivos sedativos como la trazodona, para mejorar la calidad de sueño y prevenir las recaídas (Kolla et al 2011).

En un trabajo reciente, realizado por el equipo de Roncero (2011) en una unidad de desintoxicación, se resumen los diferentes efectos sobre el sueño de diferentes substancias y en periodo de abstinencia. Los estimulantes disminuyen la sensación de necesidad sueño, en muchas ocasiones los pacientes no tienen la percepción subjetiva de alteración del sueño o sueño no reparador. La cocaína tiene un efecto euforizante sobre el ánimo, disminuye el tiempo total del sueño y altera su continuidad. Durante el periodo de abstinencia o disminución del consumo regular, aumenta el tiempo total de sueño y la somnolencia diurna.

El consumo de cannabis a dosis altas disminuye el sueño REM y el sueño de ondas lentas. El uso de cannabis también se relaciona con el aumento del número de movimientos periódicos de las extremidades durante el sueño y sueño más fragmentado. La abstinencia de cannabis reduce la calidad del sueño, aumenta la latencia y produce sueños extraños. La abstinencia después de la exposición de 4-5 cigarrillos/día, incluso tras un período corto, se ha asociado con aumento de la latencia y de la fase REM.

Los opioides producen reacciones de alertamiento dependientes de la dosis, con aumento de la actividad muscular medida en el EMG y de los movimientos corporales. EI EEG muestra un aumento de la actividad alfa, y una disminución de las fases de sueño lento profundo y del sueño REM. Los sujetos en tratamiento agudo con metadona, refieren dormir mejor. Muestran un discreto aumento del sueño lento profundo y una disminución de las frecuencias EEG rápidas durante el sueño. Durante la fase de administración crónica de metadona el sueño nocturno no esta alterado, aunque un porcentaje de pacientes refiere insomnio de conciliación y mantenimiento. Durante las primeras 3-5 semanas de abstinencia los sujetos refieren un aumento de la actividad 
onírica e insomnio. Durante la abstinencia de opiáceos en dependientes, aumentan los sueños y la fase REM y delta. Tras la retirada, se describe un periodo de insomnio de varias semanas de duración. El insomnio persistente puede provocar una recaída en el consumo de heroína.

\section{Relación Entre Los Circuitos Neuronales de Recompensa y Ciclo Vigilia/Sueño}

Estudios neuroanatómicos y electrofisiológicos han demostrado interacciones entre el sistema hipocretinérgico y los circuitos de respuesta aguda al estrés (Winsky-Sommerer et al., 2004). Asimismo, estudios en ratas demostraron que la aplicación farmacológica de Hcrt-1 era suficiente para inducir una recaída adictiva (Boutrel et al., 2005). Una literatura ya abundante demuestra que, en efecto, existe una importantísima relación entre el sistema hipocretinérgico con el sistema dopaminérgico mesocorticolímbico. También contribuyen a esta interacción con los sistemas de recompensa, los circuitos hipotalámicos adyacentes (p.ej, neuronas que contienen $\mathrm{MCH}$ (hormona concentradora de melanina), neurotensina o sensibles a leptina (lepRB).

Recientemente estudios optogenéticos en roedores han permitido establecer relaciones causales entre la actividad de circuitos neuronales y comportamientos complejos. Así, la activación optogenética selectiva del sistema hipocretinérgico, incrementa la probabilidad de la transición del sueño a la vigilia (Adamantidis el al., 2007), y también es suficiente para iniciar un comportamiento compulsivo de recaída adictiva. Como deciamos en los apartados anteriores, la literatura ha revelado numerosas asociaciones entre el abuso de drogas y deficiencias clínicas en la arquitectura del sueño. Por otra parte, varios estudios clínicos han mostrado que los pacientes narcolépticos deficientes en Hcrt, que reciben dosis frecuentes de psicoestimulantes (anfetaminas, metilfenidato o modafinil) para el tratamiento de la somnolencia diurna excesiva, son relativamente resistentes a la adicción.

El papel del sistema hipocretinérgico en la adicción sería el de actuar conjuntamente con el sistema dopaminérgico y añadir un valor positivo a la recompensa (de Lecea et al., 2006). La activación del sistema dopaminérgico puede tener consecuencias en el papel de las neuronas hipocretinérgicas durante el sueño, ya que controlan la generación del ritmo theta en el hipocampo y facilitan las transiciones sueño vigilia. Esto explicaría porqué los pacientes adictos muestran un sueño fragmentado con múltiples despertares nocturnos.

Además de la interacción Hcrt-dopamina, hay otros circuitos compartidos entre la regulación del estado de vigilia y la recompensa cerebral. En concreto, la actividad del sistema noradrénergico del locus coeruleus, esta altamente correlacionada con los estados de vigilia e inversamente proporcional al sueño (Aston-Jones y Shipley, 1987). También el sistema serotonérgico es importante para la regulación de la entrada en sueño REM, y contribuye de forma notable como neuromodulador en el estado de animo en general y en conductas adictivas
Cabe señalar que el ciclo vigilia sueño se manifiesta a través cambios de actividad globales de la corteza cerebral en el electroencefalograma. Además, los circuitos de motivación y recompensa convergen en la corteza prefrontal. Así pues también hay mecanismos de control "bottom-up" que comparten las transiciones de sueño a vigilia y las de vigilia a hipervigilia. Es de esperar que cuando se establezcan nuevas implicaciones de circuitos neuronales en el comportamiento adictivo, se revelen también nuevas asociaciones con las redes neuronales responsables de la regulación del ciclo de sueño vigilia. El mayor conocimiento de esta interacción permitiría entender mejor los mecanismos de la adicción y encontrar nuevas estrategias para el tratamiento de las adicciones (Plaza-Zabala, Maldonado y Berrendero 2012).

\section{Conflicto de intereses}

Los autores declaran no tener ningún conflicto de intereses en esta publicación.

\section{Referencias}

Adamantidis, A. R., Zhang, F., Aravanis, A. M., Deisseroth, K., y de Lecea, L. (2007) Neural substrates of awakening probed with optogenetic control of hypocretin neurons. Nature, 450, 420-424.

Aston-Jones, G. y Shipley, M. T. (1987) Response: asymmetry of neural feedback in the organization of behavioral States. Science, 237, 538.

Boutrel B., Kenny P. J., Specio, S. E., Martin-Fardon, R., Markou, A., Koob, G. F., y de Lecea, L. (2005) Role for hypocretin in mediating stress-induced reinstatement of cocaine-seeking behavior. Proceedings of the National Academy of Science U S A, 102: 19168- 19173.

Brower, K. J., Krentzman, A. y Robinson, E. A. (2011) Persistent insomnia, abstinence, and moderate drinking in alcoholdependent individuals. American Journal of Addiction, 20, 435440.

Cañellas, F., Ochogavia, J., Llobera, J., Palmer, A., Castell, J. y Iglesias, C. (1998) Trastornos del sueño y consumo de hipnóticos en la isla de Mallorca. Revista Clínica Española, 198,719-725.

de Lecea L., Kilduff, T. S., Peyron, C., Gao, X., Foye, P. E., Danielson, P. E., ... y Sutcliffe, J. G. (1998) The hypocretins: hypothalamusspecific peptides with neuroexcitatory activity. Proceedings of the National Academy of Science U SA, 95, 322-327.

de Lecea, L., Jones, B. E., Boutrel, B., Borgland, S. L., Nishino, S., Bubser, M., y DiLeone, R. (2006) Addiction and arousal: alternative roles 
of hypothalamic peptides. Journal of Neurosciences, 26, 10372-

10375

Ford, D. E. y Kamerow, D. B. (1989) Epidemiologic study of sleep disturbances and psychiatric disorders. An opportunity for prevention? Jama; 262, 1479-1484.

Hungs, M. y Mignot, E. (2001) Hypocretin/orexin, sleep and narcolepsy. Bioessays, 23, 397-408.

Irwin, M. R., Valladares, E. M., Motivala, S., Thayer, J. F. y Ehlers, C.L. (2006) Association between nocturnal vagal tone and sleep depth, sleep quality, and fatigue in alcohol dependence. Psychosomatic Medicine, 68, 159-166.

Kolla, B. P. Mansukhani, M. P., Terry y Schneekloth, T. (2011) Pharmacological Treatment of Insomnia in Alcohol Recovery: A Systematic Review. Alcohol and Alcoholism, 46, 578-585.

Roane, B. M. y Taylor, D. J. (2008). Adolescent insomnia as a risk factor for early adult depression and substance abuse. Sleep, 31, 13511356.

Roncero, C., Grau-López, L., Díaz-Morán, S., Miquel, L., Martínez-Luna, N. y Casas, M. (2012). Evaluación de las alteraciones del sueño en pacientes drogodependientes hospitalizados. Medicina Clinica, 138, 332-335.

Plaza-Zabala, A., Maldonado, R. y Berrendero, F. (2012). The Hypocretin/Orexin System: Implications for Drug Reward and Relapse. Molecular Neurobiology, 45, 424439.

Sakurai, T., Amemiya, A., Ishii, M., Matsuzaki, I., Chemelli, R. M., Tanaka, H., ... y Yanagisawa, M. (1998). Orexins and orexin receptors: a family of hypothalamic neuropeptides and $\mathrm{G}$ protein-coupled receptors that regulate feeding behavior. Cell, 92, 573-585.

Sutcliffe, J. G. y de Lecea, L. (2002). The hypocretins: setting the arousal threshold. Nature Reviews of Neurosciences, 3, 339-349.

Winsky-Sommerer, R., Yamanaka, A., Diano, S., Borok, E., Roberts, A. J., Sakurai, T. ... y de Lecea, L. (2004). Interaction between the corticotropin-releasing factor system and hypocretins (orexins): a novel circuit mediating stress response. Journal of Neurosciences, $24,1439-1448$. 